\title{
Diffuse ionized gas and its effects on nebular metallicity estimates of star-forming galaxies
}

\author{
N. Vale Asari, ${ }^{1,2 \star} \dagger$ G. S. Couto, ${ }^{3,1}$ R. Cid Fernandes, ${ }^{1}$ G. Stasińska, ${ }^{4}$ \\ A. L. de Amorim, ${ }^{1}$ D. Ruschel-Dutra, ${ }^{1}$ A. Werle,,${ }^{5,1}$ T. Z. Florido ${ }^{1}$ \\ ${ }^{1}$ Departamento de Física-CFM, Universidade Federal de Santa Catarina, C.P. 476, 88040-900, Florianópolis, SC, Brazil \\ ${ }^{2}$ School of Physics and Astronomy, University of St Andrews, North Haugh, St Andrews KY16 9SS, UK \\ ${ }^{3}$ Centro de Astronomía (CITEVA), Universidad de Antofagasta, Avenida Angamos 601, Antofagasta, Chile \\ ${ }^{4}$ LUTH, CNRS, Observatoire de Paris, PSL University, France \\ ${ }^{5}$ Instituto de Astronomia, Geofísica e Ciências Atmosféricas, Universidade de São Paulo, R. do Matão 1226, 05508-090 São Paulo, Brazil
}

Accepted .... Received 4 September 2019; in original form ...

\begin{abstract}
We investigate the impact of the diffuse ionized gas (DIG) on abundance determinations in star-forming ( $\mathrm{SF}$ ) galaxies. The DIG is characterised using the $\mathrm{H} \alpha$ equivalent width $\left(W_{\mathrm{H} \alpha}\right)$. From a set of $1409 \mathrm{SF}$ galaxies from the Mapping Nearby Galaxies at APO (MaNGA) survey, we calculate the fractional contribution of the DIG to several emission lines using high- $S / N$ data from SF spaxels (instead of using noisy emission-lines in DIG-dominated spaxels). Our method is applicable to spectra with observed $W_{\mathrm{H} \alpha} \gtrsim 10 \AA$ (which are not dominated by DIG emission). Since the DIG contribution depends on galactocentric distance, we provide DIG-correction formulae for both entire galaxies and single aperture spectra. Applying those to a sample of $>90000$ SF galaxies from the Sloan Digital Sky Survey, we find the following. (1) The effect of the DIG on strong-line abundances depends on the index used. It is negligible for the $([\mathrm{O}$ III $] / \mathrm{H} \beta) /([\mathrm{N}$ II $] / \mathrm{H} \alpha)$ index, but reaches $\sim 0.1$ dex at the high-metallicity end for $[\mathrm{N} \mathrm{II}] / \mathrm{H} \alpha$. (2) This result is based on the $\sim \mathrm{kpc}$ MaNGA resolution, so the real effect of the DIG is likely greater. (3) We revisit the mass-metallicity-star formation rate (SFR) relation by correcting for the DIG contribution in both abundances and SFR. The effect of DIG removal is more prominent at higher stellar masses. Using the $[\mathrm{N}$ II $] / \mathrm{H} \alpha$ index, $\mathrm{O} / \mathrm{H}$ increases with SFR at high stellar mass, contrary to previous claims.
\end{abstract}

Key words: galaxies: abundances - galaxies: ISM - ISM: abundances

\section{INTRODUCTION}

The diffuse ionized gas (DIG) is a warm $\left(\sim 10^{4} \mathrm{~K}\right)$, low density $\left(\sim 10^{-1} \mathrm{~cm}^{-3}\right)$ gas phase found in the interstellar medium of galaxies (e.g. Haffner et al. 2009). While early studies of this faint line-emitting component (usually referred to as the warm ionized medium in the context of our Galaxy) focused on the surroundings of the Milky Way disk (Hoyle \& Ellis 1963; Reynolds 1984), the DIG has also been observed in different galaxy structures such as extraplanar regions in edge-on disks (Boettcher et al. 2016; Levy et al. 2019), between H II regions and interarms in face-

^ email: natalia@astro.ufsc.br

$\uparrow$ Royal Society-Newton Advanced Fellowship on spirals (Greenawalt et al. 1998; Kreckel et al. 2016), and also in spheroids (bulges and ellipticals, Gomes et al. 2016). Tentative explanations for the ionization of the diffuse gas include leakage of Lyman continuum photons from star-forming regions (e.g. Weilbacher et al. 2018); photoionization by hot low-mass evolved stars (HOLMES; Stasińska et al. 2008; Flores-Fajardo et al. 2011); fast shocks from supernova winds (Dopita \& Sutherland 1995; Allen et al. 2008); turbulent dissipation (Binette et al. 2009); and heating by cosmic rays or dust grains (Reynolds \& Cox 1992). However, the properties of the DIG and how it affects physical measurements of galaxies are still debatable.

From narrow-band $\mathrm{H} \alpha$ imaging, Zurita et al. (2000) and Oey et al. (2007) estimated that the DIG contributes to 30-60 per cent of the total $\mathrm{H} \alpha$ flux in local spiral galax- 
ies. Yet the contribution from this tenuous but ubiquitous component to the emission line fluxes is generally neglected in the analysis of integrated (i.e. not spatially resolved) galaxy spectra. When estimating a galaxy's star formation rate from its $\mathrm{H} \alpha$ luminosity, for instance, one usually assumes that all the observed $\mathrm{H} \alpha$ results from photoionization by massive young stars. Similarly, estimates of the nebular oxygen abundance in star-forming (SF) galaxies neglect the contribution of the DIG to lines involved in the calculation of O/H (see Sanders et al. 2017 and references therein). Clearly, it would be useful to quantify to which extent these and other emission-line diagnostics are affected by the DIG.

Erroz-Ferrer et al. (2019), studying 38 galaxies with high spatial resolution observed with the Multi Unit Spectroscopic Explorer (MUSE; Bacon et al. 2010), found that metallicities in $\mathrm{H}$ II regions and the DIG have consistent radial distributions, but median values in $\mathrm{H}$ II regions are $\sim 0.1$ dex higher than in the DIG. Also making use of MUSE, Kumari et al. (2019) selected 24 nearby spiral galaxies to study the DIG metallicity. Defining pairs of $\mathrm{H}$ II and DIG regions that are close enough to have the same metallicity, they measure the metallicity difference of each DIG region relative to its $\mathrm{H}$ II region counterpart when applying the same strong-line calibrations. With this procedure, they propose corrections for the effect of the DIG to several metallicity indicators.

In this study, we propose a different approach. We estimate the DIG contribution by comparing the total galaxy emission in a given aperture with the emission attributed only to SF regions in that same aperture. Our analysis thus does not depend on faint emission lines typical of the DIG, but instead relies on higher signal-to-noise emission lines from either $\mathrm{SF}$ or $\mathrm{SF}+\mathrm{DIG}$ regions.

It is also important to use a reasonable criterion to define what the DIG is. Several criteria have been considered to date. For example, Blanc et al. (2019) define the DIG as zones of high $[\mathrm{S} \mathrm{II}] / \mathrm{H} \alpha$ (this method is also used by Erroz-Ferrer et al. 2019), whereas Zhang et al. (2017) define the DIG as zones of low surface brightness in $\mathrm{H} \alpha\left(\Sigma_{\mathrm{H} \alpha}\right.$; a scheme also applied by Sanders et al. 2017). A combination of $[\mathrm{S} \mathrm{II}] / \mathrm{H} \alpha$ and $\Sigma_{\mathrm{H} \alpha}$ is employed by Blanc et al. (2009), Kaplan et al. (2016) and Poetrodjojo et al. (2019), who explore criteria tailored for individual galaxies observed with integral field spectroscopy (IFS). Using IFS data from the CALIFA survey (Sánchez et al. 2012, 2016; Husemann et al. 2013; García-Benito et al. 2015), Lacerda et al. (2018, hereafter L18) define the DIG as zones of low $\mathrm{H} \alpha$ equivalent width $\left(W_{\mathrm{H} \alpha}\right)$. As argued in that paper, a definition of the DIG must be related to the total stellar population covered by the aperture. For example, galaxy bulges may have quite a high $\Sigma_{\mathrm{H} \alpha}$, yet - in galaxies devoid of active galactic nuclei (AGN) and star formation in their central parts - this zone is ionized by HOLMES (Gomes et al. 2016) and clearly corresponds to the DIG. Such zones are not identified as DIG using the $\Sigma_{\mathrm{H} \alpha}$ criterion, but are correctly identified as DIG using the $W_{\mathrm{H} \alpha}$ criterion (see fig. 1 in L18).

Applying this definition to CALIFA galaxies, L18 found systematic variations in $\mathrm{H} \alpha$ flux fractions along the Hubble sequence, ranging from a dominant DIG in ellipticals and S0 $\left(f_{\mathrm{H} \alpha}^{\mathrm{DIG}}>90\right.$ per cent), to a dominant SF component in $\mathrm{Sc}$ and later type spirals $\left(f_{\mathrm{H} \alpha}^{\mathrm{SF}}>90\right.$ per cent). Intermediate fractions are obtained for intermediate Hubble types, e.g. DIG fractions of $\sim 50$ per cent for Sb galaxies. It is worth recalling that the bimodal distribution of $W_{\mathrm{H} \alpha}$ at the root of this scheme is also seen in integrated galaxy data (Cid Fernandes et al. 2010, 2011).

In this paper we apply this same DIG-identification framework to an 8 times larger sample from the Mapping Nearby Galaxies at APO (MaNGA, Bundy et al. 2015) survey. Our central goal is to evaluate the effects of DIG emission upon line luminosities observed in integrated galaxy spectra, and then to estimate how this affects nebular metallicities obtained from strong line methods which are also applied to integrated galaxy spectra. In particular we will examine how this impacts the mass-metallicity relation $(M-$ $Z$ ), more specifically in its version where the star formation rate (SFR) acts as a second parameter, the $M-Z-S F R$ relation.

It is important to stress from the outset that our ability to correct for DIG effects is limited by the spatial resolution of the data. This limitation should nonetheless be contrasted with the case of integrated spectroscopy, where the lack of spatial information mixes different line emitting regimes into a total of inseparable components. In this context, despite of their limitations, the DIG corrections shown here represent a step forward, even if only first order corrections.

The outline of the paper is as follows. Section 2 describes the MaNGA data and how it is processed. Section 3 presents the distribution of $W_{\mathrm{H} \alpha}$ as a function of radius and host morphology, similarly to what has been done by L18 for CALIFA. Section 4 explains the procedure we propose to correct line luminosities measured from single-aperture spectra for the DIG contribution. Section 5 quantifies the bias in the metallicities obtained from various line indicators when neglecting the effect of the DIG. In Section 6 we show how contamination by DIG emission affects the relations between mass, metallicity, and SFR for SF galaxies in the seventh data release (DR7, Abazajian et al. 2009) of the Sloan Digital Sky Survey (SDSS, York et al. 2000). Section 7 summarises our main findings. Throughout this work, we assume a flat $\Lambda$ CDM cosmology with $\Omega_{0}=0.3$ and $H_{0}=70 \mathrm{~km} \mathrm{~s}^{-1} \mathrm{Mpc}^{-1}$.

\section{MANGA DATA}

MaNGA (Bundy et al. 2015) is an IFS survey from SDSS IV (Blanton et al. 2017) that will observe $\sim 10000$ galaxies across $\sim 2700 \mathrm{deg}^{2}$ of sky up to $z \sim 0.03$ from 360 to 1000 $\mathrm{nm}$ with resolution $R \sim 2000$. The observations are made with integral field units with 19 to 127 fibres of 2 arcsec in diameter, yielding a spatial sampling of $1-2 \mathrm{kpc}$. In this work, we use the 4824 currently available MaNGA galaxy datacubes from SDSS DR15 (Aguado et al. 2019). Redshifts used in this work come from the NASA-Sloan Atlas (NSA, Blanton et al. 2011) catalogue catalogue (extracted from the MaNGA drpall table version 2.4.3); 4679 datacubes have redshifts measured.

Most of the data used in this work are obtained directly from MaNGA spectra. We also use morphological classifications from the Galaxy Zoo I project (Lintott et al. 2008, 2011). The concentration index CI is taken as the ratio between the 90 and the 50 per cent Petrosian light radius (i.e. 
$R_{90}$ and $\left.R_{50}\right)$ in the $r$-band as obtained from the SDSS DR7 database (Abazajian et al. 2009).

\subsection{Preprocessing steps and measurements}

We use linearly-sampled reduced datacubes downloaded from https://data.sdss.org/sas/dr15/manga/spectro/ redux/v2_4_3/[PLATE]/stack/. The cubes were preprocessed according to the following steps:

(i) For quality assurance, we use the MASK extension from the datacubes, which contains flags for each spatial position and wavelength, and for whole spaxels. We mask spaxels with low fibre coverage or foreground stars, and we also mask bad values from the spectra (flagged as DONOTUSE).

(ii) MaNGA spectra are stored using vacuum wavelengths. Our analysis is based on data in air wavelengths. We convert vacuum to air wavelengths using the IAU standard conversion, given in Morton (1991).

(iii) The cubes are spatially resampled to $1 \operatorname{arcsec} \times 1 \operatorname{arcsec}$ pixels. This amounts to a $2 \times 2$ binning of the original $0.5 \operatorname{arcsec} \times 0.5 \operatorname{arcsec}$ sampling of the MaNGA cubes, which is an oversampling given the $\sim 2.5 \operatorname{arcsec}$ FWHM of the PSF. The fluxes are added together, and the error spectra are added in quadrature. Due to the way the cube is reconstructed in the MaNGA pipeline, spaxels are spatially correlated. To take the covariance into account, we multiply the combined errors by the factor $1+1.62 \log N$, with $N=4$, according to the recipe given by Law et al. (2016). Fluxes and errors in binned spaxels with less than 4 components, due to masked data, are scaled assuming the missing data is equal to the average of the binned spaxel. This is done separately for each wavelength. If all components are masked, the binned spaxel is masked.

(iv) After the spatial resampling, we further discard most binned spaxels with $S / N<3$ around $5635 \AA$, but keeping a few of them to 'fill in the image' using a convex hull algorithm, to avoid non-contiguous images. Next, we discard all binned spaxels where $>50$ per cent of spectral points were masked in the previous step. Three datacubes (plate-ifudsgn identifications: 8312-6101, 8317-1902 and 8332-1901) have been discarded at this step, as they contained no spaxel with $\mathrm{S} / \mathrm{N} \geqslant 3$ around $5635 \AA$. Thus only the remaining 4676 cubes have gone through the rest of the preprocessing steps described below.

(v) We correct for the the Galactic extinction using the Cardelli, Clayton \& Mathis (1989) dust law with $R_{V}=3.1$. The color index $E(B-V)$ is extracted from the FITS header. These values were obtained from Schlegel, Finkbeiner \& Davis (1998) dust maps.

(vi) The spectra are shifted to the rest frame, and resampled to the wavelength range 3600-10 400 with $\Delta \lambda=1 \AA$.

(vii) Modelling of stellar continua is done with the inverse spectral synthesis code STARLIGHT (Cid Fernandes et al. 2005). We model the observed spectrum (masking out bad pixels, emission line spectral windows, and sky features) as a sum of stellar populations attenuated by a single dust screen following the Cardelli et al. (1989) dust law with $R_{V}=3.1$.

Although the MaNGA pipeline does a fairly good job at removing the sky emission, it may fail in some cases. This is especially visible in O I 25577 and Naג5896 (sodium from street lamps). These lines are flagged as sky features to
STARLIGHT- data around these lines are kept in the resulting spectra, but are not used in the spectral fit. The emission from $\mathrm{OH}$ radicals creates a 'forest' of lines in the red part of the spectra, which can also be tricky to be removed correctly. For simplicity, we choose not to fit the spectra in wavelengths above $8900 \AA$.

The STARLIGHT fits use the 2016 updated spectral synthesis models by Bruzual \& Charlot $(2003)^{1}$, with a Chabrier (2003) initial mass function, MILES stellar library below 7351 A (Sánchez-Blázquez et al. 2006; Falcón-Barroso et al. 2011) combined with STELIB stellar library at redder wavelengths (Le Borgne et al. 2003), and 'Padova (1994)' (Alongi et al. 1993; Bressan et al. 1993; Fagotto et al. 1994a,b; Girardi et al. 1996) evolutionary tracks. Theoretical spectra from the Tlusty (Lanz \& Hubeny 2003a,b), Martins (Martins et al. 2005), UVBlue (Rodríguez-Merino et al. 2005) and PoWR (Sander et al. 2012) libraries are downgraded in spectral resolution and used to complement observed stars in the MILES stellar library. We use a base of 96 composite stellar populations obtained by assuming constant star formation rates in 16 logarithmically-spaced age bins between $1 \mathrm{Myr}$ and $14 \mathrm{Gyr}$ and 6 metallicities between $Z=0.0001$ to 0.05 (similar to the base used by Werle et al. 2019, but there they used not-yet-public updated Bruzual \& Charlot models).

(viii) We assemble all data and STARLIGHT products into a fits file for analysis within the PYCASSO software (Cid Fernandes et al. 2013; de Amorim et al. 2017).

(ix) Emission line measurements. See the following section.

\subsection{Emission line measurements}

Two codes were used to model emission lines: IFSCUBE $^{2}$ and DOBBY $^{3}$. Earlier tests with the MaNGA 14th data release cubes showed that measurements with DOBBY and IFSCUBE are consistent; in the following we thus show details and results for DOBBY.

Emission line fluxes are measured fitting Gaussian profiles to the residual spectra, which are obtained by subtracting the STARLIGHT modeled continua from the observed spectra. DOBBY uses the ASTROPY fitting model (Astropy Collaboration et al. 2013, 2018), which calls the SCIPY (Jones et al. 2019) wrapper for the MINPACK implementation (Garbow et al. 1980) of the Levenberg-Marquardt algorithm.

The fits obey a number of a priori 'common sense' physical constraints: lines from the same ion must have the same kinematics (same central velocity and intrinsic velocity dispersion) we require the $\mathrm{H} \alpha / \mathrm{H} \beta$ line flux ratio to be $\geqslant 2.6$, and the [O III] $\lambda 5007 /[\mathrm{O}$ III]4959 and [N II] $\lambda 6584 /[\mathrm{N} \mathrm{II}] 6548$ line ratios to be fixed according to the values predicted by atomic physics. Imperfections in the stellar continuum model

\footnotetext{
1 http://www.bruzual.org/ gbruzual/cb07/Updated_version_ 2016/

2 IFSCUBE is a free Python package for the analysis of integral field spectroscopy data cubes, which includes an emission line fitter (ELF), and is available at https://bitbucket.org/danielrd6/ if scube.

3 DOBBY is a free ELF available at https://bitbucket.org/ streeto/pycasso2.
} 
Table 1. Emission line continuum pass bands.

\begin{tabular}{lccc}
\hline Line & Line centre $[\AA]$ & Blue Cont. $[\AA]$ & Red Cont. $[\AA]$ \\
\hline$[\mathrm{O}$ II $] \lambda 3726$ & 3726.032 & $3653-3713$ & $3741-3801$ \\
{$[\mathrm{O}$ II $] \lambda 3729$} & 3728.815 & $3653-3713$ & $3741-3801$ \\
$\mathrm{H} \beta$ & 4861.330 & $4770-4830$ & $4890-4910$ \\
{$\left[\mathrm{O}_{\mathrm{III}}\right] \lambda 4959$} & 4958.911 & $4915-4950$ & $5015-5050$ \\
{$\left[\mathrm{O}_{\mathrm{III}}\right] \lambda 5007$} & 5006.843 & $4915-4950$ & $5015-5050$ \\
{$\left[\mathrm{~N}_{\mathrm{II}}\right] \lambda 6548$} & 6548.040 & $6480-6530$ & $6600-6670$ \\
$\mathrm{H} \alpha$ & 6562.820 & $6480-6530$ & $6600-6670$ \\
{$\left[\mathrm{~N}_{\mathrm{II}}\right] \lambda 6584$} & 6583.460 & $6480-6530$ & $6600-6670$ \\
{$\left[\mathrm{~S}_{\mathrm{II}}\right] \lambda 6716$} & 6716.440 & $6650-6700$ & $6745-6790$ \\
{$\left[\mathrm{~S}_{\mathrm{II}}\right] \lambda 6731$} & 6730.810 & $6650-6700$ & $6745-6790$ \\
\hline
\end{tabular}

are accounted for by allowing for a local pseudo-continuum in the fits, modeled as a Legendre polynomial of degree 16.

Besides emission line fluxes $(F)$ for a given emission line, we also compute equivalent widths $(W)$. The continuum flux density $C$ used to compute $W=F / C$ is evaluated with the standard procedure of calculating the median continua on side-bands and using a linear interpolation to measure the continuum at the line centre. Table 1 shows the line centres and side bands for all emission lines used in this work. We find it more convenient to measure $C$ in the STARLIGHT synthetic spectra, as it is free of noise artefacts.

The total width of the Gaussian profile is calculated by adding the intrinsic and the instrumental widths in quadrature, where the latter is taken from the dispersion spectra in the original MaNGA cubes. The noise $\sigma_{N}$ on the continuum under emission lines is taken to be the rms in the detrended residual continua in the two windows blueward and redward of the lines. The amplitude-to-noise ratio $(A / N)$ of an emission line is the Gaussian amplitude divided by $\sigma_{N}$. Following Rola \& Pelat (1994), the uncertainty in flux measurements is taken to be $\sigma_{F}=\sigma_{N} \sqrt{6 \sigma_{\lambda} \Delta \lambda}$, where $\sigma_{\lambda}$ is the Gaussian dispersion in $\AA$, and $\Delta \lambda=1 \AA$ is the spectral sampling. The signal-to-noise ratio of an emission line is thus given by $S / N=F / \sigma_{F}$.

\subsection{The MaNGA master sample}

From the 4676 fully preprocessed datacubes, we have selected only those with no observation or calibration problems (i.e. with a data quality flag DRP3QUAL $=0$, which means that no bitmask value is set on this flag, as explained in Law et al. 2016, and whose table 16 is updated online at https://www.sdss.org/dr13/algorithms/ bitmasks/\#MANGA_DRP3QUAL). We also removed repeated observations of the same galaxies, keeping only the observation made with the largest number of fibres, or, in case of a tie, the greatest exposure time. This left us with 4131 galaxies.

We have then applied the following cuts to our sample:

(i) After looking at SDSS images for galaxies in our preprocessed sample, we decided to remove very edge-on systems by selecting galaxies with an axial ratio $b / a \geqslant 0.3$ (as measured by NSA, using the column nsa_elpetro_ba from drpall).

(ii) We then removed objects whose probability of being in a merging system is $>0.4$ (Darg et al. 2010). Objects for which there is no such information in Galaxy Zoo I are also removed.

(iii) A small fraction of the remaining galaxies contain too many masked spaxels, due to the removal of foreground objects in the field. To filter out the worst cases we kept galaxies with at least 80 per cent useful spaxels (see Section 2.1) within $1 R_{50}$ from the centre of the observation (defined as the reference pixel in the data cubes).

Our final master sample of 3236 galaxies is comprised of all objects that satisfy those cuts and are tagged either as Primary+ or as Secondary MaNGA targets. This master sample is used in Section 3, where we validate the DIG identification methodology proposed by L18.

\section{THE DISTRIBUTION OF THE EQUIVALENT WIDTH OF H $\alpha$ IN MANGA SPAXELS}

Analysing data from the CALIFA survey (Sánchez et al. 2012, 2016; Husemann et al. 2013; García-Benito et al. 2015), L18 found a strongly bimodal distribution of $W_{\mathrm{H} \alpha}$ (also noted by Belfiore et al. 2016 using MaNGA data), with peaks at $\sim 1$ and $14 \AA$ and a minimum at $\sim 3 \AA$. This lead them to the following classification scheme:

- $W_{\mathrm{H} \alpha}<3 \AA$ zones trace the HOLMES-powered DIG, or hDIG. Such zones appear frequently in spheroids (throughout elliptical galaxies and also in bulges), as well as above and below disks. Their energetics, line ratios, and locations are all consistent with a HOLMES origin although a contribution from shocks is not excluded.

- $W_{\mathrm{H} \alpha}>14 \AA$ zones are associated with star-forming complexes (SFc), typical of disks. SFc trace SF sites in a galaxy, although, because of the $1-2 \mathrm{kpc}$ resolution, they cannot be considered pure $\mathrm{SF}$ regions.

- $W_{\mathrm{H} \alpha}=3-14 \AA$ zones constitute the mixed DIG (mDIG), a buffer category where line emission may be understood as a mixture of hDIG and SFc, but which probably includes other processes as well, such as photon leakage from $\mathrm{HII}$ regions, OB runaways, or shocks.

It must be noted that, while the $3 \AA$ limit corresponds to the minimum of the distribution in $W_{\mathrm{H} \alpha}$ among galaxies and is effectively closely linked to the value expected for ionisation by a purely old stellar population (see Cid Fernandes et al. 2011), the $14 \AA$ limit is arbitrary, and serves only to separate regions that contain a good proportion of $\mathrm{SF}$-powered line emission from regions whose ionization is due to a mixture of processes.

It is also important to mention that, at the $\sim \mathrm{kpc}$ resolution of MaNGA (also CALIFA), even regions classified as SFc will contain some diffuse gas emission. This is why we prefer the 'SF complex' terminology instead of the more standard 'SF region', which semantically suggests a regime where only $\mathrm{H}$ II regions contribute to the line emission.

The hDIG/mDIG/SFc classification of the nebular regime introduced in L18 is rooted on the bimodality of $W_{\mathrm{H} \alpha}$ seen in CALIFA data. The similarity with the bimodality observed in integrated galaxy spectra (Cid Fernandes et al. 2011) and the analogy of the concepts of hDIG and retired galaxy (Stasińska et al. 2008) strengthen the case for the proposed criteria, but it is the exact distribution of $W_{\mathrm{H} \alpha}$ 
in CALIFA spaxels that led to the quantitative criteria to separate the emission-line zones into $\mathrm{hDIG} / \mathrm{mDIG} / \mathrm{SFc}$. It is thus fit to start our analysis by checking whether this classification scheme also applies to MaNGA data.

Fig. 1 shows the distribution of $W_{\mathrm{H} \alpha}$ for MaNGA spaxels. As in fig. 2 of L18, the histograms are broken into ranges in distance from the nucleus $(R)$ and CI, which can be used as a proxy for galaxy morphology (Strateva et al. 2001). Vertical dashed lines at $W_{\mathrm{H} \alpha}=3$ and $14 \AA$ mark the boundaries between hDIG, mDIG, and SFc. Numbers on each panel of Fig. 1 report the fraction of the total $\mathrm{H} \alpha$ flux coming from the hDIG $\left(W_{\mathrm{H} \alpha}<3 \AA\right)$, mDIG $(3-14 \AA)$, and $\mathrm{SFc}(>14$ $\AA)$ components. We further subdivide each histogram into ranges in $[\mathrm{N} \mathrm{II}] / \mathrm{H} \alpha$ (grey histograms, which are commented later on). Here we concentrate on the full histograms (black solid line).

The top-left panel, which contains 785919 spaxels from our master sample of 3236 galaxies (from which we removed the 95429 spaxels with no $\mathrm{H} \alpha$ detection), shows the evidently bimodal distribution of $W_{\mathrm{H} \alpha}$ previously seen both in CALIFA (L18) and MaNGA (Belfiore et al. 2016), as well as in the SDSS (Bamford et al. 2008; Cid Fernandes et al. 2011). Remarkably, the peak at $W_{\mathrm{H} \alpha} \sim 1 \AA$ and the minimum at 3 $\AA$ occur at the same places in these different datasets. Stellar population analysis shows that line emission in this low$W_{\mathrm{H} \alpha}$ population is predominantly due to photoionization by HOLMES (Stasińska et al. 2008; Cid Fernandes et al. 2011). The peak of the high- $W_{\mathrm{H} \alpha}$ population occurs at $14 \AA$, close to $W_{\mathrm{H} \alpha}=16 \AA$, the value found for integrated galaxy spectra (Cid Fernandes et al. 2011), and the same value of $W_{\mathrm{H} \alpha}=14$ $\AA$ found for CALIFA (L18).

The behaviour of the $W_{\mathrm{H} \alpha}$ distribution with host morphology and distance from the nucleus is also very similar to that reported in L18. As one progresses from early to late type morphologies the histograms gradually shift from hDIG to SFc dominated, as seen on the left column of Fig. 1. Early type galaxies (large CI, second row of plots) are hDIG-dominated throughout all radii. High $W_{\mathrm{H} \alpha}$ values among these galaxies occur mostly in their central regions, and are interpretable as due to AGN. Among disk galaxies the balance between the two $W_{\mathrm{H} \alpha}$ peaks reflects the bulgeto-disk ratio, with the ionizing radiation field changing from one dominated by old stars (HOLMES) in the bulge to one dominated by OB stars in the disk. The SFc population rises both as $R$ increases and as CI decreases.

To conclude, the similarity between the results found in this section and those reported in L18 justifies the use of the same $W_{\mathrm{H} \alpha}$-based $\mathrm{hDIG} / \mathrm{mDIG} / \mathrm{SFc}$ classification scheme to MaNGA data.

As an addition to the analysis, the histograms in Fig. 1 are shaded according to the $[\mathrm{N} \mathrm{II}] / \mathrm{H} \alpha$ ratio, darker shades corresponding to smaller $[\mathrm{N} \mathrm{II}] / \mathrm{H} \alpha$. The lightest shade corresponds to a selection of spaxels whose amplitude-to-noise $\geqslant 2$ in both [N II] and $\mathrm{H} \alpha$. Darker shades draw histograms for the subsets of $A / N \geqslant 2$ spaxels where $\log [\mathrm{N}$ II $] / \mathrm{H} \alpha<-0.10$, $<-0.32,<-0.40$, i.e. the optimal transposition of the Kewley et al. (2001), Kauffmann et al. (2003) and Stasińska et al. (2006) lines from the $[\mathrm{N} \mathrm{II}] \lambda 6584 / \mathrm{H} \alpha$ versus $[\mathrm{O}$ III $] \lambda 5007 / \mathrm{H} \beta$ plane to the WHAN ([N II] $] 6584 / \mathrm{H} \alpha$ versus $\left.W_{\mathrm{H} \alpha}\right)$ diagram (Cid Fernandes et al. 2010). This shading scheme highlights the fact that regions with high $\left[\mathrm{N}_{\mathrm{II}}\right] / \mathrm{H} \alpha$ tend to have low $W_{\mathrm{H} \alpha}$, suggesting that the higher values of $[\mathrm{N} \mathrm{II}] / \mathrm{H} \alpha$ in the central zones is not a mere metallicity effect but due to ionization by HOLMES, which are more concentrated in the bulge of the galaxy than in the outskirts of the disc. Without spatially resolved spectroscopy these DIG regions would thus bias the $[\mathrm{N} \mathrm{II}] / \mathrm{H} \alpha$ ratio with respect to what one would obtain by only considering regions dominated by star formation. The following sections are dedicated to quantifying this bias and investigating its effects upon estimates of the gas phase metallicity in star-forming galaxies.

\section{HOW TO CORRECT INTEGRATED LINE LUMINOSITIES FOR THE DIG CONTRIBUTION}

We now use galaxies from our MaNGA master sample to estimate the contribution of the DIG to emission line fluxes. We start by eliminating galaxies where the presence of AGN would significantly contribute to the line emission. Photoionization models by Stasińska et al. (2006), which combined ionization by AGN and massive stars, have shown that the AGN contributes to $\lesssim 5$ per cent of the total $\mathrm{H} \alpha$ emission for objects below the Kauffmann et al. (2003) empirical demarcation line on the $[\mathrm{N} \mathrm{II}] \lambda 6584 / \mathrm{H} \alpha$ versus $[\mathrm{O}$ III] $\lambda 5007 / \mathrm{H} \beta$ plane. Therefore, selecting galaxies whose global integrated spectra are below this line, we define a SF MaNGA sample of 1409 galaxies.

Using the individual emission line measurements in each spaxel, we create several fictitious observations for the SF MaNGA galaxies by adding up individual fluxes within circular apertures of radius $R / R_{50}$. We call this total observed luminosity within the aperture $L_{\mathrm{obs}}$. We also calculate the observed equivalent width of $\mathrm{H} \alpha$ within the aperture, $W_{\mathrm{H} \alpha}^{\mathrm{obs}}$, as the ratio of $L_{\mathrm{obs}}$ and the sum of the $\mathrm{H} \alpha$ continua in those same spaxels.

For each of those $R<R_{50}$ circular apertures we add up the emission line fluxes of spaxels classified as SFc $\left(W_{\mathrm{H} \alpha} \geqslant 14 \AA\right)$, thereby removing any contribution of hDIG and $\mathrm{mDIG}$ spaxels. The luminosity of an emission line coming only from SFc spaxels is dubbed $L_{\mathrm{SFc}}$.

In the following we use $L_{\mathrm{SFc}}, L_{\mathrm{obs}}$ and $W_{\mathrm{H} \alpha}^{\mathrm{obs}}$ to guide our correction of emission line luminosities by the DIG contribution in single-aperture observations.

\subsection{Correcting entire galaxies}

In order to find a correction procedure as simple as possible, we searched for the best observational parameter with which to link the value of $f \equiv L_{\mathrm{SFc}} / L_{\mathrm{obs}}$. We experimented several easily accessible parameters measured within a circular aperture: stellar mass, the $4000-\AA$ break and $W_{\mathrm{H} \alpha}^{\mathrm{obs}}$ (where the superscript 'obs' reinforces the fact that this quantity was measured within an aperture, and is not the $W_{\mathrm{H} \alpha}$ measured in a spaxel as in Fig. 1). It turned out that $W_{\mathrm{H} \alpha}^{\mathrm{obs}}$ was the one which gave the smallest dispersion. This is not really surprising, as our definition of the DIG is closely related to $W_{\mathrm{H} \alpha}$.

Fig. 2 shows $L_{\mathrm{SFc}} / L_{\mathrm{obs}}$ as a function of $W_{\mathrm{H} \alpha}^{\mathrm{obs}}$ for [O II], $\mathrm{H} \beta,[\mathrm{O} \mathrm{III}], \mathrm{H} \alpha,\left[\mathrm{N}\right.$ II] and $\left[\mathrm{S} \mathrm{II}^{4}\right.$. The plot shows all the

${ }^{4}$ We use the following notations: $[\mathrm{O} \mathrm{II}]=[\mathrm{O} \mathrm{II}] \lambda 3726+[\mathrm{O} \mathrm{II}] \lambda 3729$, 


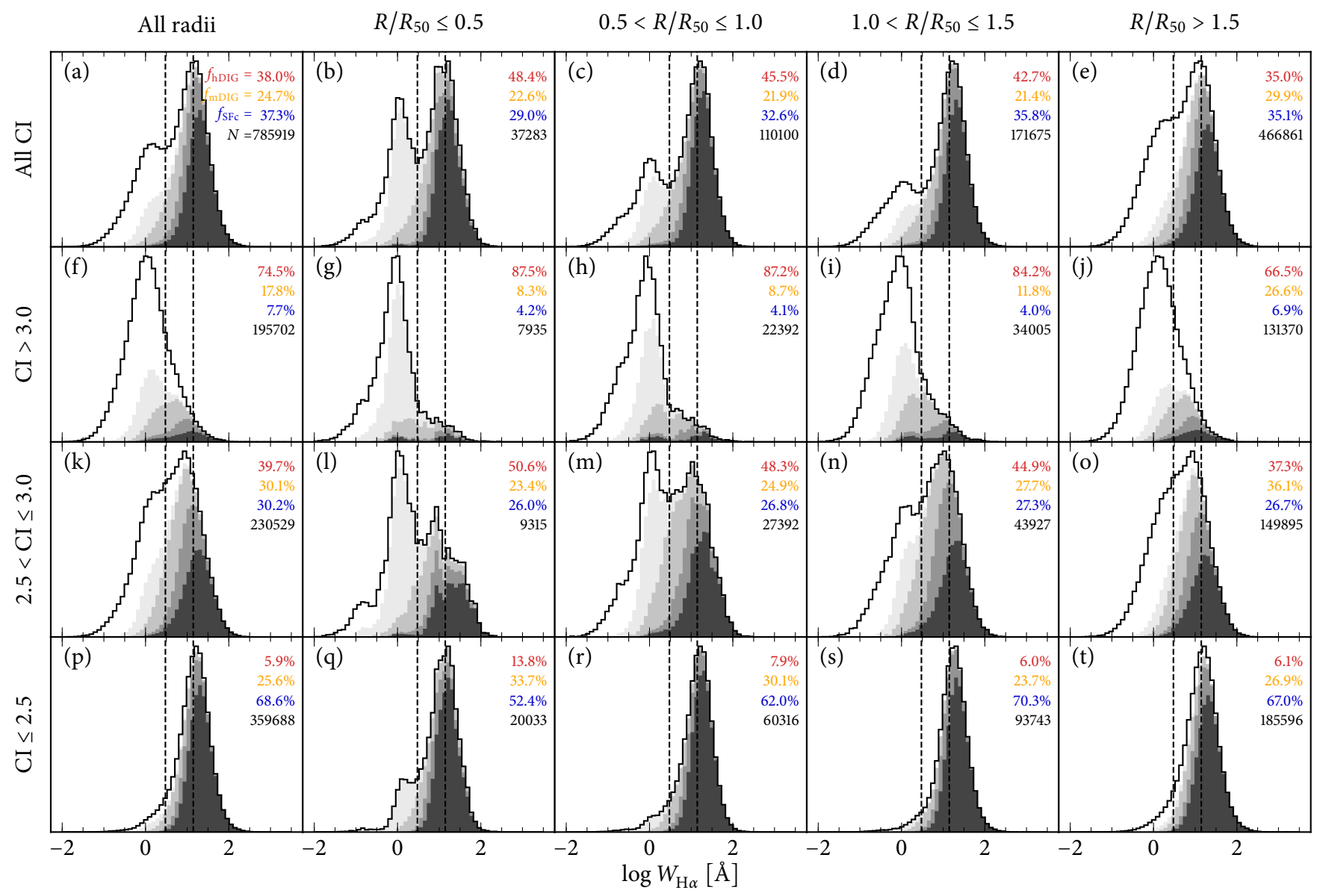

Figure 1. Distribution of $W_{\mathrm{H} \alpha}$ among 785919 spaxels of 3236 MaNGA datacubes, broken into ranges of distance to the nucleus (2nd to 5th columns) and concentration index CI (2nd to 4th rows). The top left panel shows the full sample. Panels in the top row include all host morphologies, while the left column is for all radial distances. Dashed vertical lines at 3 and $14 \AA$ mark the boundaries of the HOLMES-powered DIG (hDIG, $W_{\mathrm{H} \alpha}<3 \AA$ ), and star-forming complexes (SFc, $W_{\mathrm{H} \alpha}>14 \AA$ ), as in L18. Numbers on each panel show the average fraction of a galaxy's $\mathrm{H} \alpha$ flux coming from spaxels in the $<3,3-14$, and $>14 \AA$ ranges. The grey shading in the histograms represent cuts in $[\mathrm{N} \mathrm{II}] / \mathrm{H} \alpha$ : the lighter one is for the whole sample where $[\mathrm{N}$ II] and $\mathrm{H} \alpha$ are detected with $A / N \geqslant 2$, while the darker ones are for subsets of this $A / N \geqslant 2$ sample where $\log [\mathrm{N} \mathrm{II}] / \mathrm{H} \alpha<-0.10,-0.32$, and -0.40 .

504 galaxies for which we could calculate circular apertures of size $R / R_{50}=2.0$, i.e. which had all spaxels within $2 R_{50}$ unmasked. Note that the figure is quasi identical for any value of $R / R_{50}$ larger than $\sim 1.0$.

On each panel, the dashed line shows the fit to the data using a normal cumulative distribution function on $x=\log W_{\mathrm{H} \alpha}$ :

$f\left(\log W_{\mathrm{H} \alpha}^{\mathrm{obs}}, x_{0}, \sigma\right)=\frac{1}{\sqrt{2 \pi} \sigma} \int_{-\infty}^{\log W_{\mathrm{H} \alpha}^{\mathrm{obs}}} e^{-\left(x-x_{0}\right)^{2} / 2 \sigma^{2}} d x$.

The fitted parameters are $x_{0}$, the centre of the sloping curve, and $\sigma$, the width of the underlying Gaussian (thus a larger $\sigma$ means a shallower slope). The fitted parameters for each emission line are given in Table 2.

Using those parameters, one can correct the emission line luminosities in single-aperture spectra of entire galaxies.

$[\mathrm{S} \mathrm{II}]=[\mathrm{S} \mathrm{II}] \lambda 6716+[\mathrm{S} \mathrm{II}] \lambda 6731,[\mathrm{O} \mathrm{III}]=[\mathrm{O} \mathrm{III}] \lambda 5007$ and $[\mathrm{N} \mathrm{II}]=$ $[\mathrm{N}$ II $] \lambda 6584$.
The corrected SF emission is given by

$L_{\mathrm{SFc}}=L_{\mathrm{obs}} \frac{L_{\mathrm{SFc}}}{L_{\mathrm{obs}}}=L_{\mathrm{obs}} f\left(\log W_{\mathrm{H} \alpha}^{\mathrm{obs}}, x_{0}, \sigma\right)$.

So to correct an observed line luminosity $L_{\mathrm{obs}}$, one simply needs to choose the appropriate values of $x_{0}, \sigma$ for that given emission line and to measure $\log W_{\mathrm{H} \alpha}^{\mathrm{obs}}$. In Appendix $\mathrm{A}$, we give a sample code to correct emission line luminosities. We warn that this correction should not be applied to galaxies with $W_{\mathrm{H} \alpha} \lesssim 10 \AA$, since they are dominated by hDIG and mDIG emission (note how most curves of $L_{\mathrm{SFc}} / L_{\mathrm{obs}}$ in Fig. 2 are close to zero when $W_{\mathrm{H} \alpha}^{\mathrm{obs}}=10 \AA$ ).

A caveat to bear in mind is that, although MaNGA currently offers the largest homogeneous IFS data for this kind of study, its $\sim \mathrm{kpc}$ linear resolution limits us to identifying spaxels dominated by star forming complexes using the rather arbitrary cut at $W_{\mathrm{H} \alpha}=14 \AA$. If a statistically relevant sample of $\mathrm{SF}$ galaxies with higher resolution becomes available, those decisions and cuts should be revisited. 

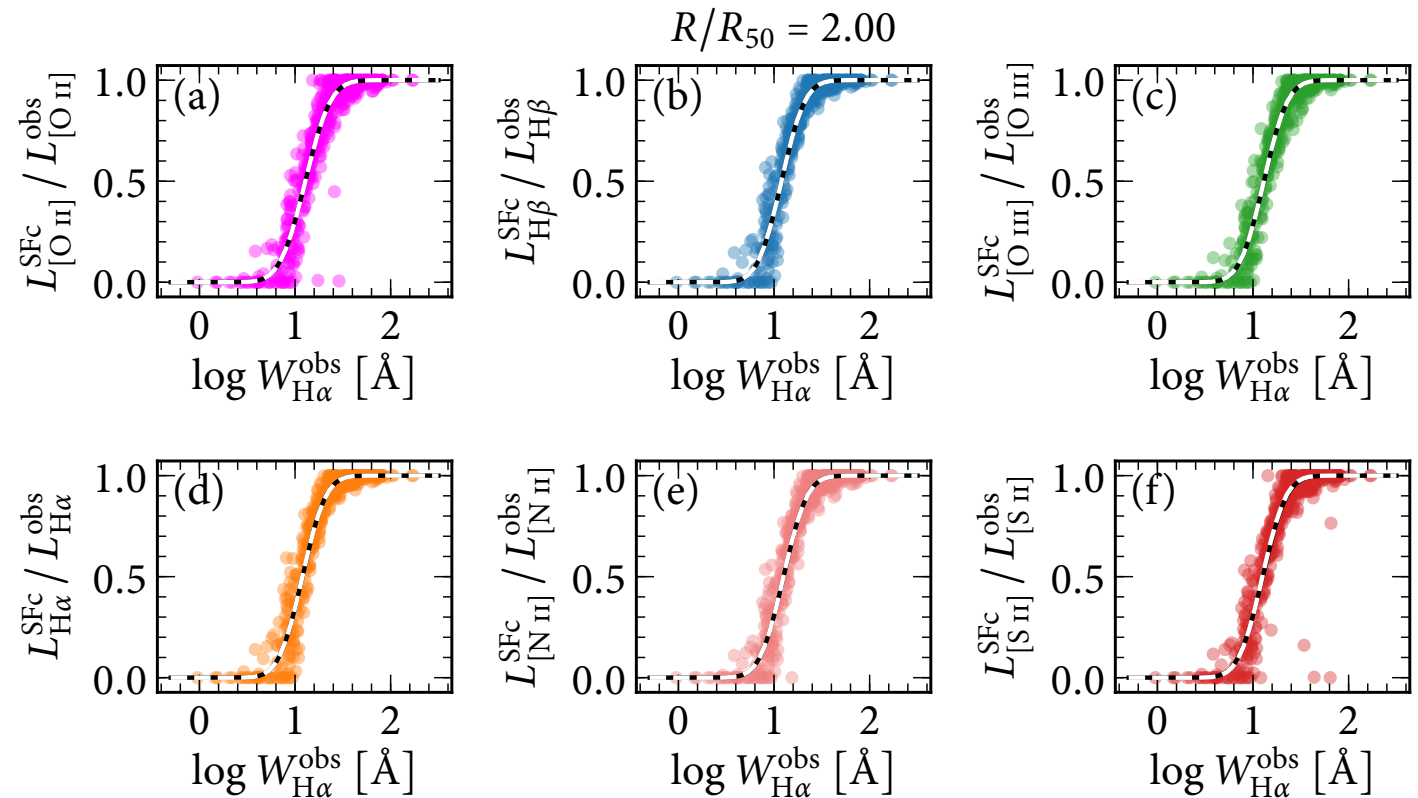

Figure 2. The ratio of the line luminosity in SFc spaxels $L_{\mathrm{SFc}}$ to the total luminosity $L_{\mathrm{obs}}$ as a function of $W_{\mathrm{H} \alpha}^{\mathrm{obs}}$. All quantities are measured in a circular aperture of size $R / R_{50}=2.0$ for 504 MaNGA SF galaxies. Each panel shows the results for a given emission line. The overimposed solid black and dashed white lines show a fit to the data using the function defined by equation 1 . This fit defines the correction to emission lines appropriate for entire galaxies, and fitted parameters are given in Table 2.

Table 2. Correction parameters suited for entire galaxies.

\begin{tabular}{lcc}
\hline Line & $x_{0}[\log \AA]$ & $\sigma[\log \AA]$ \\
\hline$[\mathrm{O}$ II $] \lambda 3726+3729$ & 1.108 & 0.2086 \\
$\mathrm{H} \beta$ & 1.078 & 0.1944 \\
{$[\mathrm{O}$ III $] \lambda 5007$} & 1.113 & 0.1963 \\
$\mathrm{H} \alpha$ & 1.077 & 0.1932 \\
{$[\mathrm{~N} \mathrm{II}] \lambda 6584$} & 1.098 & 0.1944 \\
{$[\mathrm{~S} \mathrm{II}] \lambda 6716+6731$} & 1.100 & 0.1993 \\
\hline
\end{tabular}

Table 3. Correction parameters suited for single-aperture observations of central parts of galaxies.

\begin{tabular}{lcc}
\hline Line & $x_{0}[\log \AA]$ & $\sigma[\log \AA]$ \\
\hline$[\mathrm{O} \mathrm{II}] \lambda 3726+3729$ & 1.130 & 0.1302 \\
$\mathrm{H} \beta$ & 1.121 & 0.1341 \\
{$[\mathrm{O} \mathrm{III}] \lambda 5007$} & 1.131 & 0.1262 \\
$\mathrm{H} \alpha$ & 1.121 & 0.1340 \\
{$[\mathrm{~N}$ II $] \lambda 6584$} & 1.131 & 0.1300 \\
{$[\mathrm{~S} \mathrm{II}] \lambda 6716+6731$} & 1.130 & 0.1316 \\
\hline
\end{tabular}

\subsection{Accounting for aperture effects}

As shown in L18 and also in Fig. 1, the importance of the DIG varies with the galactocentric distance. It is thus expected that, in single aperture observations, the corrections for the DIG should depend on the covering fraction of the aperture. We therefore repeated the procedure from Section 4.1 for various values of $R / R_{50}$.

It turns out that the fitted curves for $f\left(\log W_{\mathrm{H} \alpha}^{\mathrm{obs}}, x_{0}, \sigma\right)$ change very little from $R / R_{50}=0.4$ to 0.8 . We thus show in Fig. 3 only the case corresponding to $R / R_{50}=0.7$. Table 3 gives the corresponding values of $x_{0}$ and $\sigma$ for each emission line.

In the next two sections, we apply these corrections to a sample of SDSS galaxies suitable for emission-line abundance analyses.

\section{EFFECT OF THE DIG ON METALLICITY ESTIMATES IN SDSS GALAXIES}

\subsection{The SDSS DR7 sample}

We select galaxies observed by the SDSS DR7 (Abazajian et al. 2009) from the STARLiGHT database ${ }^{5}$ (Cid Fernandes et al. 2005, 2009). We apply a flux renormalisation correction to match the fibre and spectral $r$-band photometries using the spectofibre factor provided by the MPA-JHU team ${ }^{6}$. The stellar populations models used are from Bruzual \& Charlot (2003) as described in Asari et al. (2007). The stellar mass $M$ is calculated from the STARLIGHT spectral synthesis modelling as in Cid Fernandes et al. (2005), corrected for the mass outside the fibre using the $z$-band photometry. Line luminosities are measured in the residual spectra after removing the stellar continua from the observed spectra using the method detailed in Mateus et al. (2006); Stasińska et al. (2006).

We start from a sample of 777967 galaxies similar to the one described by Koziel-Wierzbowska et al. (2017): we select all galaxies from the Main Galaxy Sample (Strauss

\footnotetext{
5 The public database can be accessed at http://starlight. ufsc.br.

6 http://www.mpa-garching.mpg.de/SDSS/DR7/raw_data.html
} 

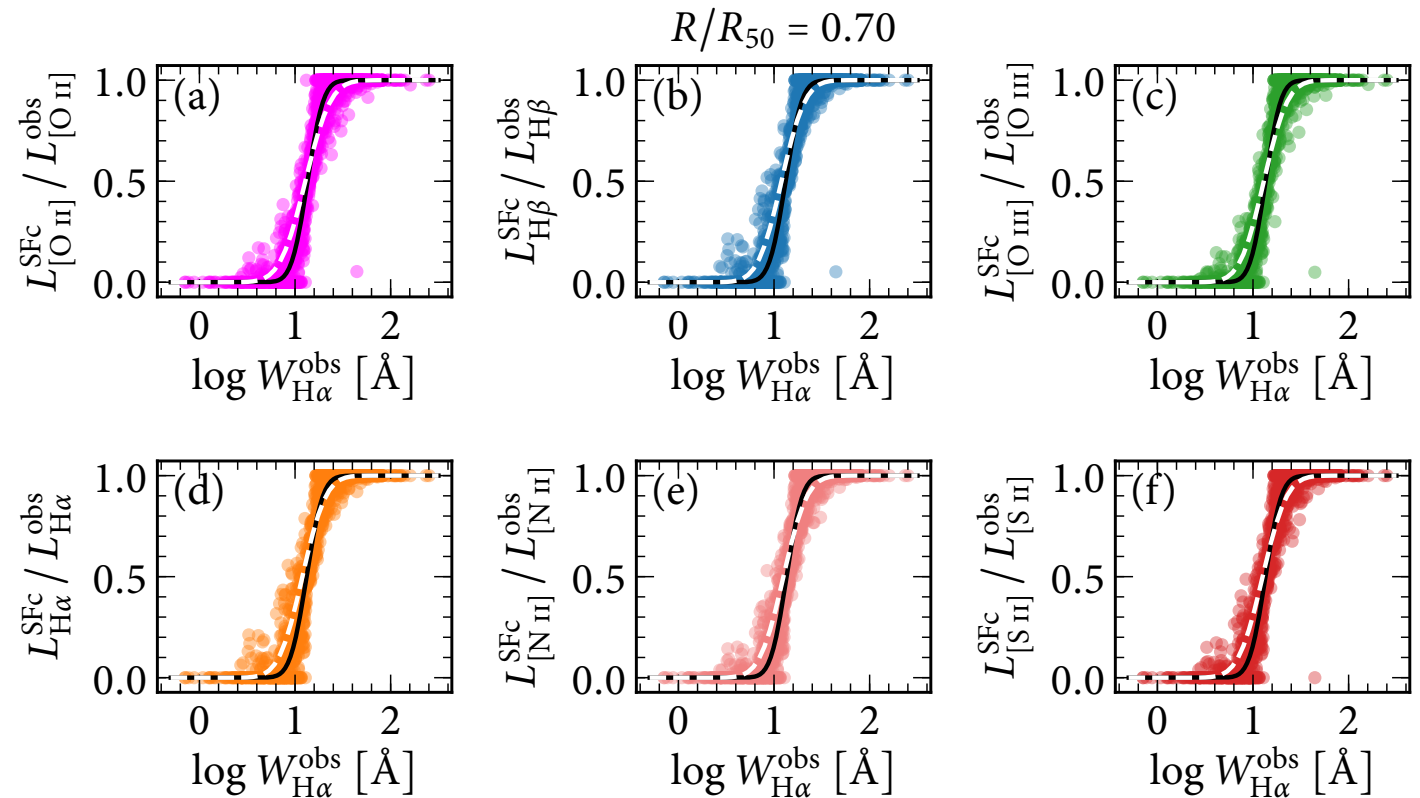

Figure 3. As Fig. 2, but for a circular aperture of size $R / R_{50}=0.7$ for 1409 MaNGA SF galaxies. The solid black line is a fit to those measurements, and the dashed white line repeats the fit for $R / R_{50}=2.0$ from Fig. 2 for comparison. The corrections shown in those plots are appropriate for central observations of galaxies, such as those in the SDSS DR7, and fitted parameters are given in Table 3.

et al. 2002) and the Luminous Red Galaxy (Eisenstein et al. 2001) catalogues; we impose a minimum signal-to-noise of 5 at the $4020 \AA$ continuum, and we remove galaxies with $M \leqslant 10^{7} \mathrm{M}_{\odot}$ or with negative values of $R_{50}$.

From this starting sample, we obtain a sample of 94335 $\mathrm{SF}$ galaxies by applying the following criteria. Inspired by Mannucci et al. (2010), we select objects (1) below the Kauffmann et al. (2003) line on the $[\mathrm{N}$ II $] \lambda 6584 / \mathrm{H} \alpha$ versus [O III] $\lambda 5007 / \mathrm{H} \beta$ plane, (2) which have a minimum $S / N$ of 25 in their $\mathrm{H} \alpha$ line detection, (3) with nebular $A_{V}<2.5$ (calculated from the Balmer decrement using a Cardelli et al. (1989) extinction law with $R_{V}=3.1$ ), and (4) with redshifts limited to the $0.07 \leqslant z \leqslant 0.30$ range. We also impose other common sense cuts: we remove very edge-on systems by selecting $b / a \geqslant 0.3$, and remove $\mathrm{hDIG} / \mathrm{mDIG}$-dominated spectra by leaving only those with $W_{\mathrm{H} \alpha} \geqslant 10 \AA$.

\subsection{Metallicity biases}

We use the recent Curti et al. (2017) calibrations to estimate the gas-phase oxygen abundances $(\mathrm{O} / \mathrm{H})$ from three strong line indices: $\mathrm{N} 2, \mathrm{O} 23$ and $\mathrm{O} 3 \mathrm{~N} 2^{7}$, which are valid in the $12+\log \mathrm{O} / \mathrm{H}=7.60-8.85,8.40-8.85$, and 7.60-8.85 ranges, respectively. We also use the Dopita et al. (2016) calibration of $\mathrm{O} / \mathrm{H}$ as a function of $\mathrm{N} 2$ and $\mathrm{N} 2 \mathrm{~S} 2^{8}$ (their equations 1 and 2 , valid in the $12+\log \mathrm{O} / \mathrm{H}=8.00-9.00$ range), and call it D16. We correct all the relevant emission lines to calculate each index, and for $\mathrm{O} 23$ we apply a reddening correction to

7 We use the notation $\mathrm{N} 2$ for $\left[\begin{array}{ll}\mathrm{N} & \mathrm{II}\end{array}\right] \lambda 6584 / \mathrm{H} \alpha$, O23 for $\left(\left[\begin{array}{ll}\mathrm{O} & \mathrm{III}\end{array}\right] \lambda 5007+4959+\left[\begin{array}{ll}\mathrm{O} & \mathrm{II}\end{array}\right] \lambda 3726+3729\right) / \mathrm{H} \beta-$ which is called R23 by Curti et al. (2017) -, and O3N2 for $([\mathrm{O} \mathrm{III}] \lambda 5007 / \mathrm{H} \beta) /([\mathrm{N} \mathrm{II}] \lambda 6584 / \mathrm{H} \alpha)$.

$8 \mathrm{~N} 2 \mathrm{~S} 2$ is for $[\mathrm{N} \mathrm{II}] \lambda 6584 /[\mathrm{S} \mathrm{II}] \lambda 6716+6731$. all lines using the $\mathrm{H} \alpha / \mathrm{H} \beta$ Balmer decrement and a Cardelli et al. (1989) dust law with $R_{V}=3.1$.

Fig. 4 compares $\mathrm{O} / \mathrm{H}$ obtained from the observed emission lines to $\mathrm{O} / \mathrm{H}$ calculated from the emission lines after applying the correction for DIG emission assuming a covering fraction $R / R_{50}=0.7$, thus applicable to SDSS observations of galaxies up to redshifts of $\sim 0.5$ (which is roughly the redshift limit for objects in the Luminous Red Galaxy sample of SDSS DR7).

It can be seen that the strongest biases are found at the highest metallicities. O3N2 is the least affected abundance indicator, since both $[\mathrm{O}$ III $] \lambda 5007 / \mathrm{H} \beta$ and $[\mathrm{N} \mathrm{II}] \lambda 6584 / \mathrm{H} \alpha$ rise almost in tandem in the DIG (as obvious from the position of DIG and $\mathrm{SFc}$ spaxels in the $[\mathrm{O} \mathrm{III}] / \mathrm{H} \beta$ versus $[\mathrm{N}$ II $] / \mathrm{H} \alpha$ diagram, e.g. see fig. 9 of L18). Kumari et al. (2019) also concluded that O3N2 is the abundance indicator that is the least biased by the DIG. The N2 index is the most affected by the DIG, especially at high metallicity, leading to an overestimate of $\mathrm{O} / \mathrm{H}$ by up to 0.1 dex if no correction is made.

The points in Fig. 4 have been colour-coded according to their value of $W_{\mathrm{H} \alpha}^{\mathrm{obs}}$, as indicated in the colour scale on the bottom right. As expected, it is those galaxies with the smallest values of $W_{\mathrm{H} \alpha}^{\mathrm{obs}}$ which show the largest abundance bias. The fact that galaxies with high metallicities are also the ones with low $W_{\mathrm{H} \alpha}^{\text {obs }}$ is related to those objects having both a larger DIG emission and a smaller specific SFR (due to the mass-metallicity relation and the decrease in specific SFR with increasing stellar mass, e.g. Kauffmann et al. 2003). Moreover, the low $W_{\mathrm{H} \alpha}^{\text {obs }}$-high $\mathrm{O} / \mathrm{H}$ (thus high stellar mass) relation is also in part due to observational selection effects: at a given value of $W_{\mathrm{H} \alpha}^{\mathrm{obs}}$, galaxies with the highest stellar mass are brighter and more easily detectable. Since we are simply showing the effect of the DIG to O/H esti- 

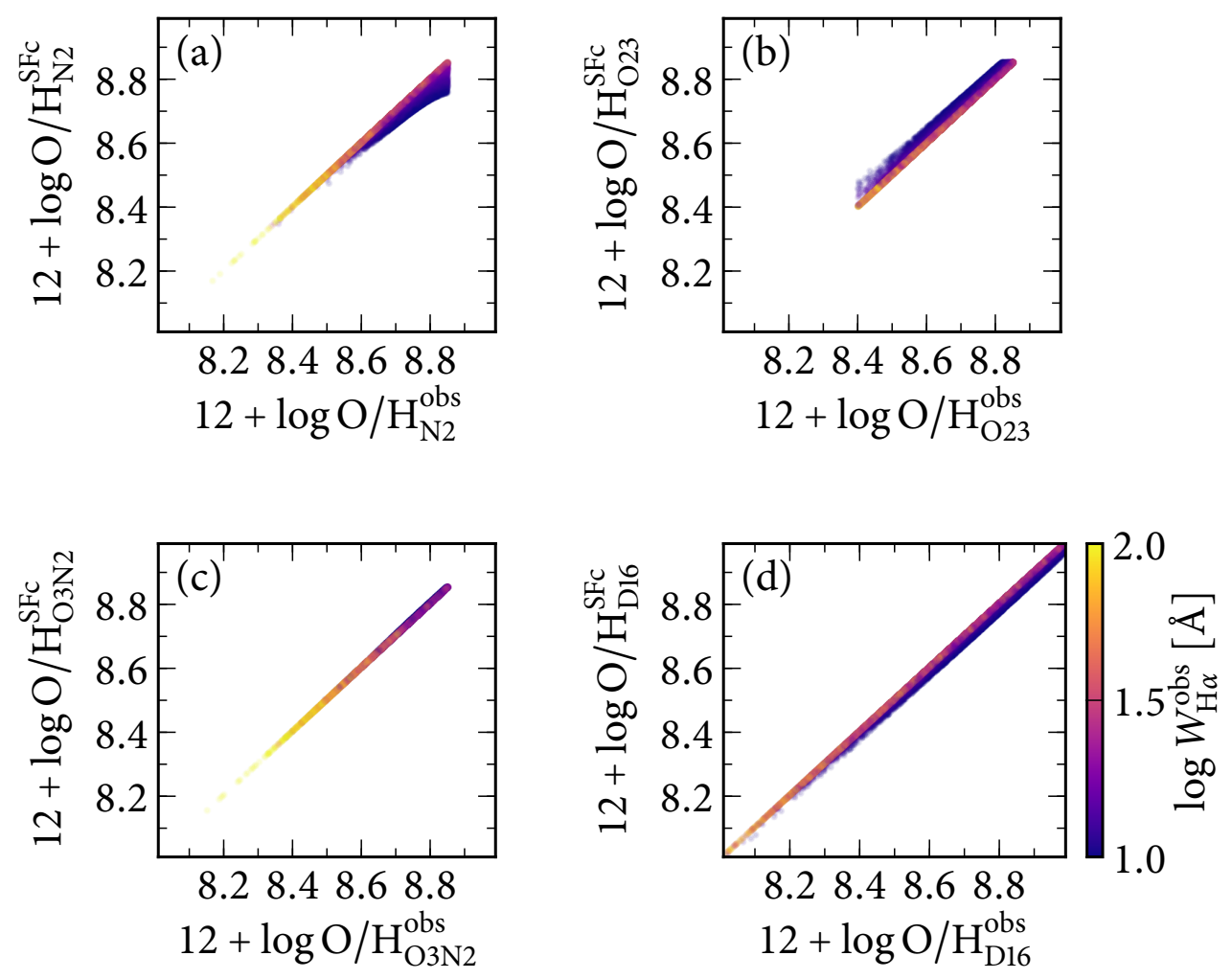

Figure 4. Comparison of nebular metallicity estimates for 94335 SF galaxies from the SDSS DR7. The abscissa shows metallicities calculated using emission lines with no correction, whereas the ordinate is after removing the DIG emission using the correction appropriate for SDSS-like apertures. Each panel shows a different calibration applied with different indices: N2, O23 and O3N2 (using calibrations from Curti et al. 2017) and D16 (by Dopita et al. 2016, whose index is a combination of N2 and N2S2). Points are colour-coded by their $W_{\mathrm{H} \alpha}$, to highlight where the DIG correction is more important.

mates, these selection effects are not key to this paper. Note that, for higher signal-to-noise observations, which will be obtained for surveys using larger telescopes than the one used for SDSS, a larger proportion of galaxies with low values of $W_{\mathrm{H} \alpha}^{\mathrm{obs}}$ will be present, because the condition of a high signal-to-noise in the emission lines will be more easily reached. Thus, the average abundance bias will be larger than for the SDSS survey.

\section{EFFECT OF THE DIG ON THE $M-Z-$ SFR RELATION}

The dependence of the $M-Z$ relation on the SFR is not yet clear. Previous studies using integrated spectra showed that nearby galaxies in a fixed stellar mass bin show an anticorrelation between $Z$ and SFR at low values of $M$ (Ellison et al. 2008; Lara-López et al. 2010a,b; Mannucci et al. 2010), suggesting e.g. inflow of low- $Z$ gas to feed SF in the galaxies, or outflows of enriched gas. Sanchez Almeida \& Sanchez-Menguiano (2019), using a sample of 736 nearby spiral galaxies with MaNGA spatially resolved data, suggested that the global $M-Z-S F R$ relation is a result of the local anti-correlation between the SFR surface density and the gas metallicity. Nevertheless, other studies, using spatially resolved data and various metallicity indicators, could not find such relation between the $M-Z$ and SFR, indicating that the relation may arise from e.g. an aperture effect in single-fibre spectroscopy (Sánchez et al. 2017) or how the $M$-bins are defined (Barrera-Ballesteros et al. 2017). The $M-$ $Z$-SFR relation also depends on the choice of the metallicity indicator, as demonstrated by Kashino et al. (2016) and as can be seen in Fig. 5 below. Making use of the calibration proposed by Dopita et al. (2016), those authors showed that the anti-correlation between metallicity and SFR disappears for the lowest masses.

All things considered, correctly estimating the gasphase oxygen abundances of galaxies is crucial in the analysis of such relations. As mentioned above, the DIG contribution to the line emission is not negligible and will affect the metallicity indicators if not taken into account.

Fig. 5 shows the SFR versus $\mathrm{O} / \mathrm{H}$ in bins of stellar mass, as in fig. 1 of Mannucci et al. (2010). Each row shows a different estimate of $\mathrm{O} / \mathrm{H}$ using the four indices from the last section. On the left we show the plots where $\mathrm{O} / \mathrm{H}$ and SFR have been obtained without any DIG correction. In the middle, we show the same quantities, but now correcting the determination of $\mathrm{O} / \mathrm{H}$ for the presence of DIG. On the right 


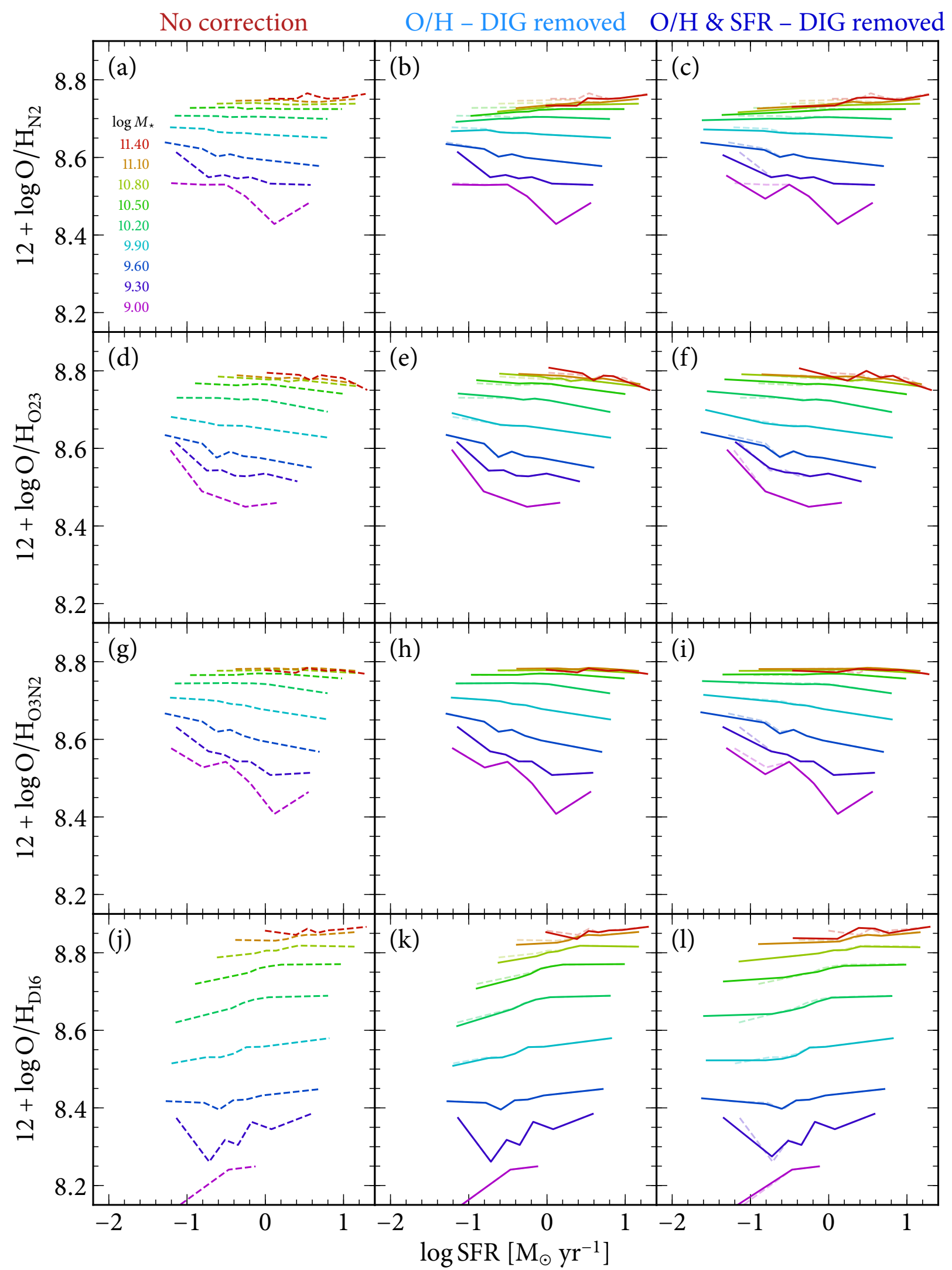

Figure 5. Each panel shows $\mathrm{O} / \mathrm{H}$ as a function of SFR in 0.30-dex-wide stellar mass bins for a sample of 94335 SF galaxies from the SDSS DR7. Panel (a) shows the $\log M$ centre values for each bin, and also the colour-coding for each median curve. Rows show results for the four different $\mathrm{O} / \mathrm{H}$ calibrations discussed in Sec. 5.2. The column of panels on the right shows the observed relation (dashed lines); the middle corrects the emission lines for the $\mathrm{O} / \mathrm{H}$ computation by the DIG emission (solid lines); and the right column also corrects the SFR for the DIG contribution (solid lines). The 'no correction' relation shown on the left column as dashed lines is repeated on the two other columns for comparison. 
we again show the same quantities, but now correcting both $\mathrm{O} / \mathrm{H}$ and SFR for the presence of DIG. The dashed lines from the left panels are repeated in the middle and right panels, which allows one to compare more accurately the 'no correction' with the 'DIG removed' results.

The SFR is calculated from the $\mathrm{H} \alpha$ luminosity from equation (8) of Asari et al. (2007), which was calibrated for solar metallicity and a Chabrier (2003) IMF between 0.1 and $100 \mathrm{M}_{\odot}$ using Bruzual \& Charlot (2003) stellar population models:

$\mathrm{SFR}=2 \mathrm{M}_{\odot} \mathrm{yr}^{-1}\left(\frac{L_{\mathrm{H} \alpha}}{10^{8} \mathrm{~L}_{\odot}}\right)$.

The underlying assumptions under this conversion are that (1) the SFR is constant over the lifetime of massive ionizing stars ( $10 \mathrm{Myr}),(2)$ the nebulae are ionization-bounded, i.e. no ionizing photons escape, (3) the case B recombination applies, (4) all $\mathrm{H} \alpha$ luminosity is produced by the ionization of massive stars, i.e. it ignores other ionization sources such as HOLMES.

The effect of the DIG on the SFR estimate is to increase the SFR with respect to its real value because some of the $\mathrm{H} \alpha$ emission is attributed to star formation while it is actually due to other ionization sources (such as HOLMES). This effect is stronger for the most massive galaxies, where a significant fraction of the $\mathrm{H} \alpha$ emission is not due to star formation.

As regards the effect of the $\mathrm{DIG}$ on $\mathrm{O} / \mathrm{H}$ in Fig. 5, it seems negligible when using the $\mathrm{O} 3 \mathrm{~N} 2$ or the $\mathrm{O} 23$ indices (although in the latter case this index is almost independent of metallicity for $12+\log \mathrm{O} / \mathrm{H}$ between 8.0 and 8.4 , see e.g. Curti et al. 2017). The effect however becomes noticeable when using the D16 index, and even more so when using N2. In the latter case, $\mathrm{O} / \mathrm{H}$ becomes correlated with SFR for high mass galaxies instead of being flat, because the correction is greater at smaller values of $W_{\mathrm{H} \alpha}$ (which corresponds to smaller SFR at a given mass).

We must note that the DIG-corrected abundances depend on the indices used to derive them - especially at the highest metallicities and lowest SFRs. This indicates that the strong line calibrations we used are slightly biased, probably precisely because they did not consider the influence of the DIG. This issue needs to be further investigated.

Note that the effects found in this paper could actually be much greater, and could also affect low-mass galaxies, as one may notice on the right column of Fig. 5, mainly regarding N2 and O3N2 indices. To check this we would need higher resolution data, say MUSE-like observations for the same set of MaNGA galaxies. One should beware, however, that data with too high resolution should also be used with care, since the reasoning behind the $W_{\mathrm{H} \alpha} \mathrm{hDIG} / \mathrm{mDIG} / \mathrm{SFc}$ classification is founded on the fact that the ionizing source and the ionized gas are observed on the same spaxel.

The correction for DIG using $W_{\mathrm{H} \alpha}$ for MaNGA galaxies is however physically motivated. As argued by $\mathrm{L} 18, W_{\mathrm{H} \alpha}$ is linked to the ionizing stellar population, ranging from $1 \AA$ for HOLMES to several hundred $\AA$ for ionizing young populations. $W_{\mathrm{H} \alpha}$ is also an intensive quantity, i.e. observing two hDIG regions into the same line of sight yields a resulting $W_{\mathrm{H} \alpha}$ which is characteristic of hDIG regions. This is in contrast to a cut in $\mathrm{H} \alpha$ surface brightness, which is an extensive quantity: two DIG-like regions overimposed in the same line of sight will have a higher $\Sigma_{\mathrm{H} \alpha}$ and may cross into the SFc territory. This can be especially important for bulges in the central parts of galaxies, exactly the parts observed by SDSS. As a matter of curiosity, we have tested how a cut in $\Sigma_{\mathrm{H} \alpha}$ would affect the $M-Z-\mathrm{SFR}$ relation. A simple cut like the one proposed by Zhang et al. (2017) results in similar shifts in the $M-Z-S F R$ for all calibrations, but affecting all mass bins. The only major difference to our results is an overall upward shift in D16 metallicities. We have also tested hybrid methods, which employ DIG removal criteria based on $\Sigma_{\mathrm{H} \alpha}$ and $[\mathrm{S} \mathrm{II}] / \mathrm{H} \alpha$, such the ones proposed by Kaplan et al. (2016) and Poetrodjojo et al. (2019). The results are very similar to the ones with a simple $\Sigma_{\mathrm{H} \alpha}$, but much noisier. Since the $\Sigma_{\mathrm{H} \alpha}$ and/or [S II]/H $\alpha$ cuts change from galaxy to galaxy, we did not find a global parameter which would be tightly correlated to the emission line correction factors. Cuts in $[\mathrm{S} \mathrm{II}] / \mathrm{H} \alpha$ also run against the logic of this work, which is quantifying the impact of the DIG on emission line fluxes, and propagating it to line ratios used as indices for metallicity calibrations: using a limit in $[\mathrm{S}$ II $] / \mathrm{H} \alpha$ could be equivalent to forcing a solution from the outset for indices based on $[\mathrm{S} \mathrm{II}] / \mathrm{H} \alpha$ itself.

There is an added complication that we have not dealt with: the original $M-Z-S F R$ relations (Ellison et al. 2008; Lara-López et al. 2010a,b; Mannucci et al. 2010) were found for $\mathrm{O} / \mathrm{H}$ and SFR computed using line luminosities measured inside the fibre. For $\mathrm{O} / \mathrm{H}$, since metallicity gradients tend to be very small, an aperture correction may be minimal. For the SFR, one might be able to correct to the total SFR using a method similar to Brinchmann et al. (2004). Yates, Kauffmann \& Guo (2012), for instance, have found that the median total SFR is about 0.6 dex higher than the fibre SFR, with the effect being larger for low-mass (thus less concentrated), low-redshift galaxies. Correcting the SFR in a satisfactory manner for aperture effects is however out of the scope of this work, since any estimate (either trying to account for $\mathrm{H} \alpha$ outside the fibre or correcting the integrated spectrum for the light outside the fibre and modelling the stellar population synthesis to obtain the SFR, as outlined in section 5 of Asari et al. 2007) would be severely affected by dust variations from one region to the other within a galaxy (Vale Asari et al., in preparation).

\section{SUMMARY}

The presence of the DIG has long been suspected to affect integrated spectra of galaxies. In this paper, we have focused on the effect of the DIG on abundance determinations in emission-line galaxies.

Defining the DIG according to $W_{\mathrm{H} \alpha}$, as shown to be appropriate by L18, we have used a set of $1409 \mathrm{SF}$ galaxies from the IFS MaNGA survey to find what percentage of the various emission lines luminosities is due to the DIG. Our method relies on high-S/N data from SFc spaxels, instead of relying on measurements of emission line in noisier, DIGdominated spaxels.

We have thus proposed a method to recover the line luminosities arising from $\mathrm{SFc}$ regions alone for galaxies whose observed $W_{\mathrm{H} \alpha}$ are larger than about $10 \AA$ (galaxies or galaxy zones with $W_{\mathrm{H} \alpha}$ smaller than such a value are dominated by $\mathrm{mDIG} / \mathrm{hDIG}$ emission and cannot be treated as star-forming 
galaxies). Since the importance of the DIG depends on the galactocentric distance, we propose two sets of corrections: one for integrated spectra of entire galaxies, whose number is bound to increase drastically with the development of deep and ultra-deep surveys; and another for single aperture observations of galaxies in the local universe, such as the SDSS DR7.

We apply this DIG correction to a sample of $94335 \mathrm{SF}$ galaxies from the SDSS DR7. Among the indices usually used to estimate the oxygen abundance, it is N2 which is the most affected by the presence of the DIG, producing a bias of 0.1 dex in $\mathrm{O} / \mathrm{H}$ at the highest metallicity and lowest $W_{\mathrm{H} \alpha}$. On the other hand, O3N2 is not much affected (in agreement with Kumari et al. 2019), whereas O23 and D16 are somewhat biased.

We have then revisited the $M-Z-S F R$ relation after correcting for the DIG on both $\mathrm{O} / \mathrm{H}$ and SFR. The effect is noticeable for the highest stellar masses and metallicities, and may result in $\mathrm{O} / \mathrm{H}$ increasing with $\mathrm{SFR}$ at high stellar mass (especially when using N2). This effect of course is based on a correction made with IFS data with 1-2 kpc resolution, so it should be taken as a cautionary stepping stone. The $M-$ $Z$-SFR relation might have changed more dramatically if we had at hand a large dataset of higher-resolution IFS data, and the effect might have been noticeable even for low-mass galaxies.

Note that the so-called fundamental $M-Z-$ SFR relation of Mannucci et al. (2010), which was obtained ignoring the DIG and taking the average of two metallicity indices, was also based on SDSS galaxies with $\mathrm{S} / \mathrm{N}$ in $\mathrm{H} \alpha$ larger than 25. This criterion removes preferentially massive galaxies $\left(\log M \gtrsim 11 \mathrm{M}_{\odot}\right)$. A $M-Z-\mathrm{SFR}$ relation based on selection criteria less affected by observing conditions may look quite different. Also, $Z$ and SFR were obtained with line measurements inside a spectral fibre. An aperture correction can be very tricky if one wants to take into account line luminosity variations within a galaxy (Vale Asari et al., in preparation) and outside the fibre, which is especially important to correct the SFR. Attempts to tackle this issue have been made by other authors (e.g. Yates et al. 2012).

Given how important the $M-Z-S F R$ relation is for galaxy chemical evolution models, all those selection, observational, and analysis caveats should be taken into account when comparing this empirical relation to models (e.g. Yates et al. 2012; Kashino et al. 2016). The changes brought about by the removal of the DIG contamination reveal that all assumptions underlying this relation must be carefully considered.

\section{ACKNOWLEDGEMENTS}

We are thankful to the referee, Brent Groves, for the useful comments. NVA acknowledges support of the Royal Society and the Newton Fund via the award of a Royal SocietyNewton Advanced Fellowship (grant NAF $\backslash R 1 \backslash 180403$ ), and of Fundação de Amparo à Pesquisa e Inovação de Santa Catarina (FAPESC) and Conselho Nacional de Desenvolvimento Científico e Tecnológico (CNPq). This study was financed in part by the Coordenação de Aperfeiçoamento de Pessoal de Nível Superior - Brasil (CAPES) - Finance Code 001. NVA, RCF, GS, ALA, AW and TZF ac- knowledge the support from the CAPES CSF-PVE project 88881.068116/2014-01. GSC acknowledges the support by the Comite Mixto ESO-Chile and the DGI at University of Antofagasta. AW acknowledges financial support from Fundação de Amparo à Pesquisa do Estado de São Paulo (FAPESP) process number 2019/01768-6. ALA and DRD acknowledge the financial support from Programa Nacional de Pós Doutorado (PNPD/CAPES). RCF acknowledges financial support from CNPq. The authors thank Vivienne Wild, Gustavo Bruzual and Stéphane Charlot for their help with the correct references for the updated spectral synthesis models.

Funding for the SDSS and SDSS-II has been provided by the Alfred P. Sloan Foundation, the Participating Institutions, the National Science Foundation, the U.S. Department of Energy, the National Aeronautics and Space Administration, the Japanese Monbukagakusho, the Max Planck Society, and the Higher Education Funding Council for England. The SDSS Web Site is http://www.sdss.org/. The SDSS is managed by the Astrophysical Research Consortium for the Participating Institutions. The Participating Institutions are the American Museum of Natural History, Astrophysical Institute Potsdam, University of Basel, University of Cambridge, Case Western Reserve University, University of Chicago, Drexel University, Fermilab, the Institute for Advanced Study, the Japan Participation Group, Johns Hopkins University, the Joint Institute for Nuclear Astrophysics, the Kavli Institute for Particle Astrophysics and Cosmology, the Korean Scientist Group, the Chinese Academy of Sciences (LAMOST), Los Alamos National Laboratory, the Max-Planck-Institute for Astronomy (MPIA), the Max-Planck-Institute for Astrophysics (MPA), New Mexico State University, Ohio State University, University of Pittsburgh, University of Portsmouth, Princeton University, the United States Naval Observatory, and the University of Washington.

Funding for the Sloan Digital Sky Survey IV has been provided by the Alfred P. Sloan Foundation, the U.S. Department of Energy Office of Science, and the Participating Institutions. SDSS-IV acknowledges support and resources from the Center for High-Performance Computing at the University of Utah. SDSS-IV is managed by the Astrophysical Research Consortium for the Participating Institutions of the SDSS Collaboration including the Brazilian Participation Group, the Carnegie Institution for Science, Carnegie Mellon University, the Chilean Participation Group, the French Participation Group, HarvardSmithsonian Center for Astrophysics, Instituto de Astrofísica de Canarias, The Johns Hopkins University, Kavli Institute for the Physics and Mathematics of the Universe (IPMU) / University of Tokyo, the Korean Participation Group, Lawrence Berkeley National Laboratory, Leibniz Institut für Astrophysik Potsdam (AIP), Max-Planck-Institut für Astronomie (MPIA Heidelberg), Max-Planck-Institut für Astrophysik (MPA Garching), Max-Planck-Institut für Extraterrestrische Physik (MPE), National Astronomical Observatories of China, New Mexico State University, New York University, University of Notre Dame, Observatário Nacional / MCTI, The Ohio State University, Pennsylvania State University, Shanghai Astronomical Observatory, United Kingdom Participation Group, Universidad Nacional Autónoma de México, University of Arizona, University 
of Colorado Boulder, University of Oxford, University of Portsmouth, University of Utah, University of Virginia, University of Washington, University of Wisconsin, Vanderbilt University, and Yale University.

This research made use of Astropy, ${ }^{9}$ a communitydeveloped core Python package for Astronomy (Astropy Collaboration et al. 2013, 2018).

\section{REFERENCES}

Abazajian K. N., et al., 2009, ApJS, 182, 543

Aguado D. S., et al., 2019, ApJS, 240, 23

Allen M. G., Groves B. A., Dopita M. A., Sutherland R. S., Kewley L. J., 2008, ApJS, 178, 20

Alongi M., Bertelli G., Bressan A., Chiosi C., Fagotto F., Greggio L., Nasi E., 1993, A\&AS, 97, 851

Asari N. V., Cid Fernandes R., Stasińska G., Torres-Papaqui J. P., Mateus A., Sodré L., Schoenell W., Gomes J. M., 2007, MNRAS, 381, 263

Astropy Collaboration et al., 2013, A\&A, 558, A33

Astropy Collaboration et al., 2018, AJ, 156, 123

Bacon R., et al., 2010, in Ground-based and Airborne Instrumentation for Astronomy III. p. 773508, doi:10.1117/12.856027

Bamford S. P., Rojas A. L., Nichol R. C., Miller C. J., Wasserman L., Genovese C. R., Freeman P. E., 2008, MNRAS, 391, 607

Barrera-Ballesteros J. K., Sánchez S. F., Heckman T., Blanc G. A., The MaNGA Team 2017, ApJ, 844, 80

Belfiore F., et al., 2016, MNRAS, 461, 3111

Binette L., Flores-Fajardo N., Raga A. C., Drissen L., Morisset C., 2009, ApJ, 695, 552

Blanc G. A., Heiderman A., Gebhardt K., Evans II N. J., Adams J., 2009, ApJ, 704, 842

Blanc G. A., Lu Y., Benson A., Katsianis A., Barraza M., 2019, ApJ, 877, 6

Blanton M. R., Kazin E., Muna D., Weaver B. A., Price-Whelan A., 2011, AJ, 142, 31

Blanton M. R., et al., 2017, AJ, 154, 28

Boettcher E., Zweibel E. G., Gallagher III J. S., Benjamin R. A., 2016, ApJ, 832, 118

Bressan A., Fagotto F., Bertelli G., Chiosi C., 1993, A\&AS, 100, 647

Brinchmann J., Charlot S., White S. D. M., Tremonti C., Kauffmann G., Heckman T., Brinkmann J., 2004, MNRAS, 351, 1151

Bruzual G., Charlot S., 2003, MNRAS, 344, 1000

Bundy K., et al., 2015, ApJ, 798, 7

Cardelli J. A., Clayton G. C., Mathis J. S., 1989, ApJ, 345, 245

Chabrier G., 2003, PASP, 115, 763

Cid Fernandes R., Mateus A., Sodré L., Stasińska G., Gomes J. M., 2005, MNRAS, 358, 363

Cid Fernandes R., et al., 2009, in Revista Mexicana de Astronomia y Astrofisica Conference Series. pp 127-132

Cid Fernandes R., Stasińska G., Schlickmann M. S., Mateus A., Vale Asari N., Schoenell W., Sodré L., 2010, MNRAS, 403, 1036

Cid Fernandes R., Stasińska G., Mateus A., Vale Asari N., 2011, MNRAS, 413, 1687

Cid Fernandes R., et al., 2013, A\&A, 557, A86

Curti M., Cresci G., Mannucci F., Marconi A., Maiolino R., Esposito S., 2017, MNRAS, 465, 1384

Darg D. W., et al., 2010, MNRAS, 401, 1043

Dopita M. A., Sutherland R. S., 1995, ApJ, 455, 468

9 Astropy Python package: http://www.astropy.org
Dopita M. A., Kewley L. J., Sutherland R. S., Nicholls D. C., 2016, Ap\&SS, 361, 61

Eisenstein D. J., et al., 2001, AJ, 122, 2267

Ellison S. L., Patton D. R., Simard L., McConnachie A. W., 2008, ApJ, 672, L107

Erroz-Ferrer S., et al., 2019, MNRAS, 484, 5009

Fagotto F., Bressan A., Bertelli G., Chiosi C., 1994a, A\&AS, 104, 365

Fagotto F., Bressan A., Bertelli G., Chiosi C., 1994b, A\&AS, 105, 29

Falcón-Barroso J., Sánchez-Blázquez P., Vazdekis A., Ricciardelli E., Cardiel N., Cenarro A. J., Gorgas J., Peletier R. F., 2011, A\&A, 532, A95

Flores-Fajardo N., Morisset C., Stasińska G., Binette L., 2011, MNRAS, 415, 2182

Garbow B., Hillstrom K., More J., 1980, Implementation guide for MINPACK-1. [Package of Fortran subprograms for solution of systems of nonlinear equations]. Technical Report ANL-80-68, Argonne National Lab., IL (USA), doi:10.2172/5171554

García-Benito R., et al., 2015, A\&A, 576, A135

Girardi L., Bressan A., Chiosi C., Bertelli G., Nasi E., 1996, A\&AS, 117, 113

Gomes J. M., et al., 2016, A\&A, 588, A68

Greenawalt B., Walterbos R. A. M., Thilker D., Hoopes C. G., 1998, ApJ, 506, 135

Haffner L. M., et al., 2009, Reviews of Modern Physics, 81, 969

Hoyle F., Ellis G. R. A., 1963, Australian Journal of Physics, 16,

Husemann B., et al., 2013, A\&A, 549, A87

Jones E., Oliphant T., Peterson P., et al., 2001-2019, SciPy: Open source scientific tools for Python, http://www.scipy.org/

Kaplan K. F., et al., 2016, MNRAS, 462, 1642

Kashino D., Renzini A., Silverman J. D., Daddi E., 2016, ApJ, 823, L24

Kauffmann G., et al., 2003, MNRAS, 346, 1055

Kewley L. J., Dopita M. A., Sutherland R. S., Heisler C. A., Trevena J., 2001, ApJ, 556, 121

Kozieł-Wierzbowska D., Vale Asari N., Stasińska G., Sikora M., Goettems E. I., Wójtowicz A., 2017, ApJ, 846, 42

Kreckel K., Blanc G. A., Schinnerer E., Groves B., Adamo A., Hughes A., Meidt S., 2016, ApJ, 827, 103

Kumari N., Maiolino R., Belfiore F., Curti M., 2019, MNRAS, 485,367

Lacerda E. A. D., et al., 2018, MNRAS, 474, 3727

Lanz T., Hubeny I., 2003a, ApJS, 146, 417

Lanz T., Hubeny I., 2003b, ApJS, 147, 225

Lara-López M. A., Bongiovanni A., Cepa J., Pérez García A. M., Sánchez-Portal M., Castañeda H. O., Fernández Lorenzo M., Pović M., 2010a, A\&A, 519, A31

Lara-López M. A., et al., 2010b, A\&A, 521, L53

Law D. R., et al., 2016, AJ, 152, 83

Le Borgne J.-F., et al., 2003, A\&A, 402, 433

Levy R. C., et al., 2019, arXiv e-prints,

Lintott C. J., et al., 2008, MNRAS, 389, 1179

Lintott C., et al., 2011, MNRAS, 410, 166

Mannucci F., Cresci G., Maiolino R., Marconi A., Gnerucci A., 2010, MNRAS, 408, 2115

Martins L. P., González Delgado R. M., Leitherer C., Cerviño M., Hauschildt P., 2005, MNRAS, 358, 49

Mateus A., Sodré L., Cid Fernandes R., Stasińska G., Schoenell W., Gomes J. M., 2006, MNRAS, 370, 721

Morton D. C., 1991, ApJS, 77, 119

Oey M. S., et al., 2007, ApJ, 661, 801

Poetrodjojo H., D’Agostino J. J., Groves B., Kewley L., Ho I. T., Rich J., Madore B. F., Seibert M., 2019, MNRAS, 487, 79

Reynolds R. J., 1984, ApJ, 282, 191

Reynolds R. J., Cox D. P., 1992, ApJ, 400, L33 
Rodríguez-Merino L. H., Chavez M., Bertone E., Buzzoni A., 2005, ApJ, 626, 411

Rola C., Pelat D., 1994, A\&A, 287, 676

Sanchez Almeida J., Sanchez-Menguiano L., 2019, arXiv e-prints,

Sánchez-Blázquez P., et al., 2006, MNRAS, 371, 703

Sánchez S. F., et al., 2012, A\&A, 538, A8

Sánchez S. F., et al., 2016, A\&A, 594, A36

Sánchez S. F., et al., 2017, MNRAS, 469, 2121

Sander A., Hamann W.-R., Todt H., 2012, A\&A, 540, A144

Sanders R. L., Shapley A. E., Zhang K., Yan R., 2017, ApJ, 850, 136

Schlegel D. J., Finkbeiner D. P., Davis M., 1998, ApJ, 500, 525

Stasińska G., Cid Fernandes R., Mateus A., Sodré L., Asari N. V., 2006, MNRAS, 371, 972

Stasińska G., Vale Asari N., Cid Fernandes R., Gomes J. M., Schlickmann M., Mateus A., Schoenell W., Sodré Jr. L., 2008, MNRAS, 391, L29

Strateva I., et al., 2001, AJ, 122, 1861

Strauss M. A., et al., 2002, AJ, 124, 1810

Weilbacher P. M., et al., 2018, A\&A, 611, A95

Werle A., Cid Fernandes R., Vale Asari N., Bruzual G., Charlot S., Gonzalez Delgado R., Herpich F. R., 2019, MNRAS, 483, 2382

Yates R. M., Kauffmann G., Guo Q., 2012, MNRAS, 422, 215

York D. G., et al., 2000, AJ, 120, 1579

Zhang K., et al., 2017, MNRAS, 466, 3217

Zurita A., Rozas M., Beckman J. E., 2000, A\&A, 363, 9

de Amorim A. L., Vale Asari N., 2019, Emission line flux correction due to DIG, doi:10.5281/zenodo.3378041, https://doi. org/10.5281/zenodo. 3378041

de Amorim A. L., et al., 2017, MNRAS, 471, 3727 


\section{APPENDIX A: SAMPLE PYTHON CODE TO CORRECT EMISSION LINE FLUXES}

The sample code below (also available in eletronic format at https://doi.org/10.5281/zenodo.3378041; de Amorim \& Vale Asari 2019) shows how to implement the line flux correction in Python, and use it to calculate the corrected [N II] $66584 / \mathrm{H} \alpha$ line ratio. We will assume the data come from single-aperture observations.

First, we define the error function, as given by Eq. 1. We also define dictionaries containing the coefficients for both apertures.

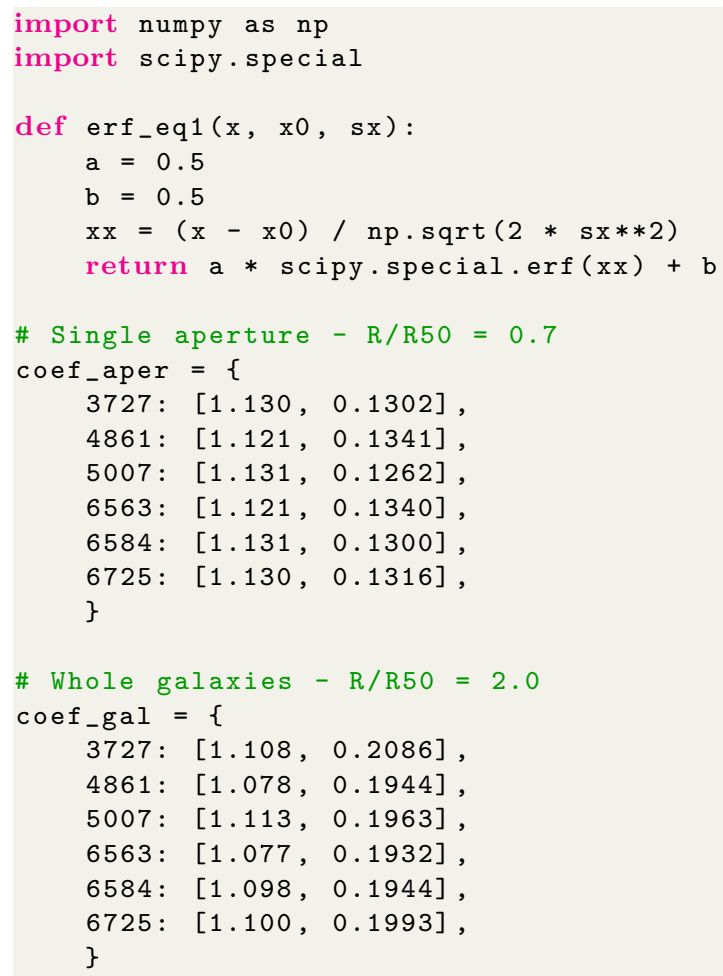

Listing 1: Initial definitions.

The user has to provide the emission line flux measurements, as well as $W_{\mathrm{H} \alpha}$. For this example, let's generate some random emission line flux and $W_{\mathrm{H} \alpha}$ measurements.

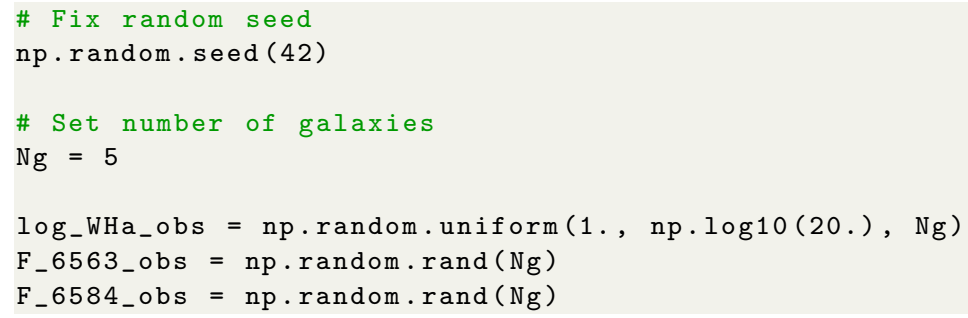

Listing 2: Generation of random data.

Here, $W_{\mathrm{H} \alpha}$ is in the $10-20 \AA$ range and the fluxes are in the $0-1$ range (the units are irrelevant in this example). For single-aperture observations, we use the coefficients defined in coef_aper. The correction, then, is done as follows. The print statements show the observed and corrected [N II] $\lambda 6584 / \mathrm{H} \alpha$ line ratios.

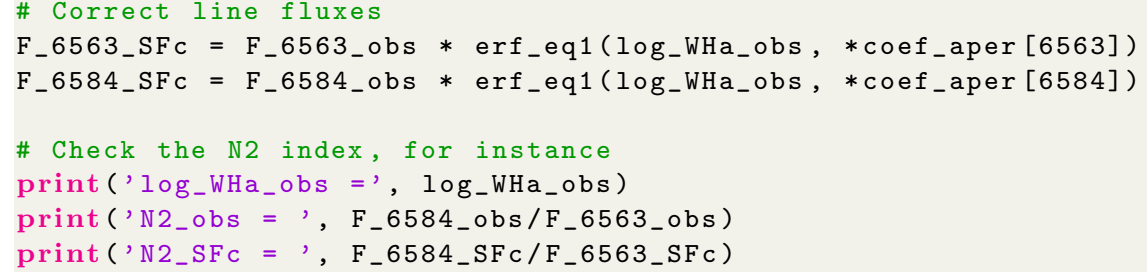

Listing 3: Flux correction. 